\title{
Convergence of finite elements enriched with mesh-less methods ${ }^{\star}$
}

\author{
Sonia Fernández-Méndez, Pedro Díez, Antonio Huerta \\ Departament de Matemàtica Aplicada III, E.T.S. de Ingenieros de Caminos, Uni- \\ versitat Politècnica de Catalunya, Campus Nord UPC, E-08034 Barcelona, Spain. \\ e-mail: antonio.huerta@upc.es
}

The date of receipt and acceptance will be inserted by the editor

Summary A combined hierarchical approximation based on finite elements and mesh-less methods is proposed and studied. Finite Elements are enriched adding hierarchical shape functions based on a particle distribution. Convergence results are presented and proved.

Key words Adaptivity, $h-p$ refinement, finite element method, mesh-less method, hierarchical interpolation, convergence, error bound, a priori estimates.

* Grant sponsor: Ministerio de Ciencia y Tecnología. Grant number: REN20010925-C03-01 


\section{Introduction}

Particle methods such as reproducing kernel particle methods (RKPM) [19,17], element-free Galerkin (EFG) [4,6,5,7,22,25], partition of unity finite element method (PUFEM) [23,1], h-p cloud method [10,11], or smooth particle hydrodynamics (SPH) [24,26,8,9], among others (see

\section{$[3,18]$ for a general presentation), have proven their applicability in} computational mechanics. They do not require to generate a mesh and thus, they are specially suited for certain problems, for instance adaptive refinement computations or discontinuous field problems
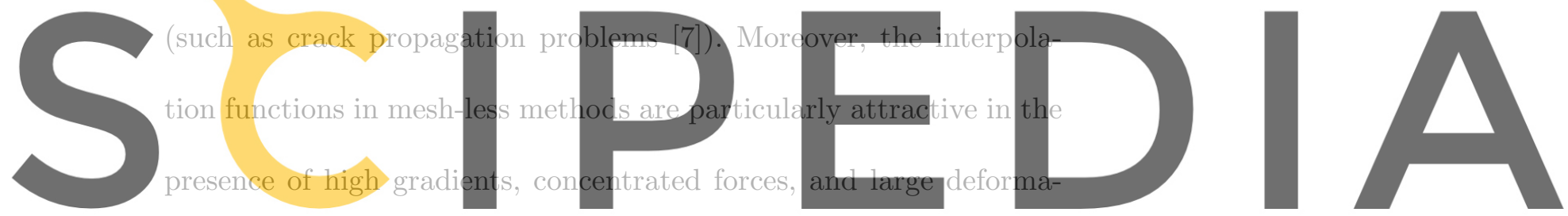

tions. Two different discretization methods are employed with these Register for free at https//www.scipedia.com to download the version without the watermark

mesh-less techniques: the classical collocation method and a Galerkin

type formulation. Choosing one or the other is, among other things, a trade off between convergence properties and computational speed.

Collocation methods can run one order of magnitude faster than finite elements but present poor rates of convergence and may present spatial instabilities. Galerkin formulations, on the other hand, obtain at least similar rates of convergence of finite elements but suffer from an important computational cost $[2,9,16]$. From a practical point of 
view, finite elements implement Dirichlet boundary conditions in a simple way and are widely used and trusted by practitioners. However, the relative cost of the mesh generation process is, for some problems, very large. In particular, the cost of remeshing in adaptive refinement problems is clearly not negligible.

In order to take advantage of both methods, many authors have proposed combined interpolations combining finite elements and particles (see $[6,13,12,21])$. In [21], Liu et al. suggest to enrich the finite element approximation with particles. The goal is to define an effec-

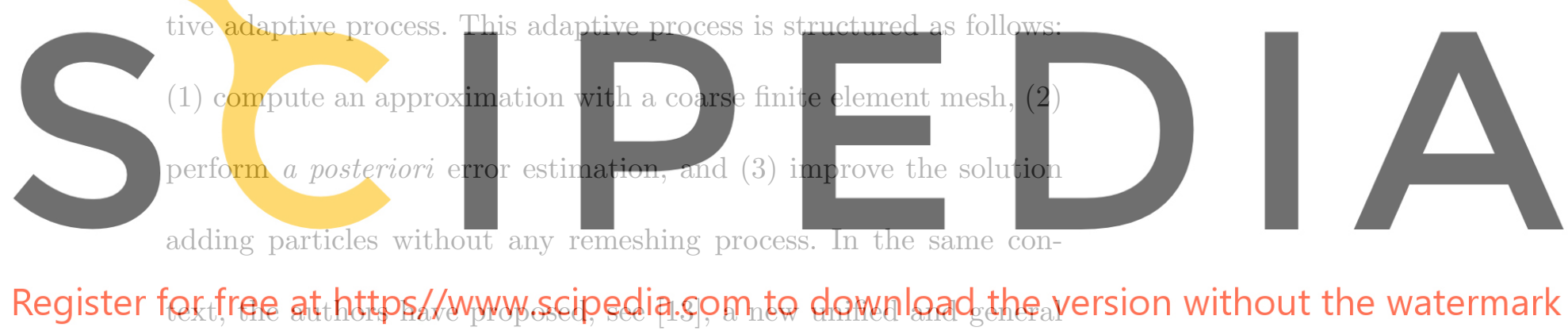

formulation for a hierarchical combined interpolation. The combined

finite element and particle interpolation is presented in two cases: coupling and enrichment. The convergence analysis for the second case is presented here, that is, the $h-p$ enrichment of finite elements with particles.

In this paper, an a priori error estimate for such a combined method is presented. A proof of this sharp error estimate is devel- 
oped under certain reasonable assumptions on the size of the finite elements. If these assumptions are not fulfilled, a less sharp error estimate is also found. This a priori error estimate is required to study convergence of the combined method and also to design proper adaptive strategies.

\section{How to enrich FEM with particles}

Let $u(x)$ be a function to be interpolated. First, $u(x)$ is approximated using a finite element mesh with nodes $\left\{x_{i}\right\}_{i \in I^{h}}$. Thus, the interpola-

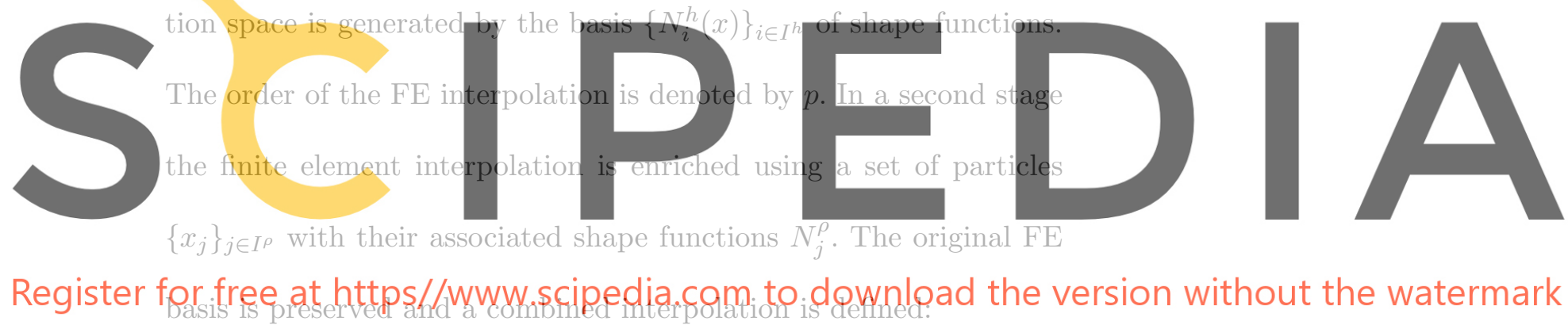

$$
u(x) \simeq u^{h}(x)+u^{\rho}(x),
$$

where

$$
u^{h}(x)=\sum_{i \in I^{h}} u\left(x_{i}\right) N_{i}^{h}(x)
$$

is the usual finite element contribution and where

$$
u^{\rho}(x)=\sum_{j \in I^{\rho}} u\left(x_{j}\right) N_{j}^{\rho}(x)
$$


is the contribution of the particles. The enrichment due to the added particles may increase the order of consistency of the interpolation. The goal of this section is to define the shape functions $N_{j}^{\rho}$ associated to each particle in order to obtain the desired consistency.

In the context of the EFG method $[4,6,5,7,22,25]$, the interpolation functions are usually defined as

$$
N_{j}^{\rho}(x)=\mathbf{P}^{T}\left(\frac{x-x_{j}}{\rho}\right) \boldsymbol{\alpha}(x) \phi\left(\frac{x-x_{j}}{\rho}\right),
$$

where $\phi$ is a window function with compact support $(\phi(z)=0$ for

$|z|>1$, i.e. $N_{j}^{\rho}(x)=0$ for $\left.\left|x-x_{j}\right|>\rho\right)$, and $\rho$ is the dilation param-

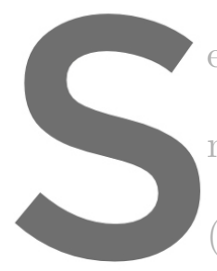

eter. The vector $\alpha$ is

mial basis $\mathbb{P}(x)=\{p$

(usually $p_{i}(x)=x^{i}$ in $1 \mathrm{D}$ and
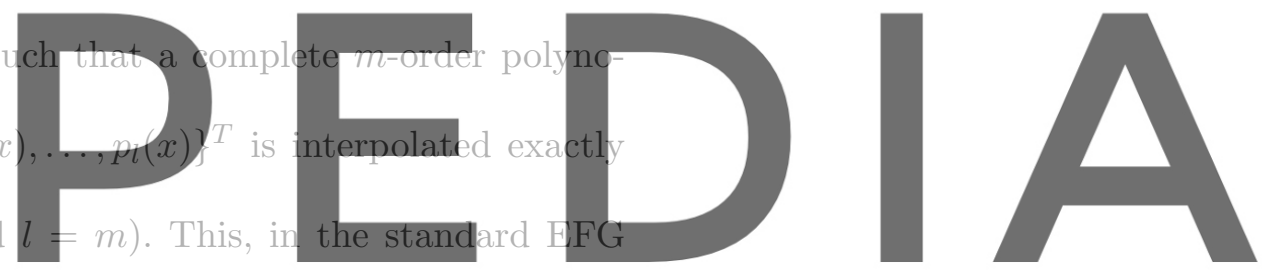

Register for free at https//Www.scipedia.com to download the version without the watermark

[15]. When the combined interpolation (2.1) is used, the m-order con-

sistency condition reads

$$
\mathbf{P}(0)=\sum_{i \in I^{h}} \mathbf{P}\left(\frac{x-x_{i}}{\rho}\right) N_{i}^{h}(x)+\sum_{j \in I^{\rho}} \mathbf{P}\left(\frac{x-x_{j}}{\rho}\right) N_{j}^{\rho}(x)
$$

It is easy to verify that, when the dilation parameter $\rho$ is constant, the previous consistency condition is equivalent to

$$
\mathbf{P}(x)=\sum_{i \in I^{h}} \mathbf{P}\left(x_{i}\right) N_{i}^{h}(x)+\sum_{j \in I^{\rho}} \mathbf{P}\left(x_{j}\right) N_{j}^{\rho}(x) .
$$


which shows more clearly the desired reproducibility condition. After (2.4) is replaced in (2.5), the following linear system of equations for $\boldsymbol{\alpha}$ is obtained

$$
\mathbf{M}(x) \boldsymbol{\alpha}(x)=\mathbf{P}(0)-\sum_{i \in I^{h}} \mathbf{P}\left(\frac{x-x_{i}}{\rho}\right) N_{i}^{h}(x),
$$

where $\mathbf{M}(x)$ is the Gram matrix

$$
\mathbf{M}(x)=\sum_{j \in I \rho} \mathbf{P}\left(\frac{x-x_{j}}{\rho}\right) \mathbf{P}^{T}\left(\frac{x-x_{j}}{\rho}\right) \phi\left(\frac{x-x_{j}}{\rho}\right) .
$$

Remark 2.1 The Gram matrix $\mathbf{M}$ is identical to the matrix employed

in the standard EFG method. Thus, as in EFG, the number of par-

ticles, their position and their related dilation parameters cannot be
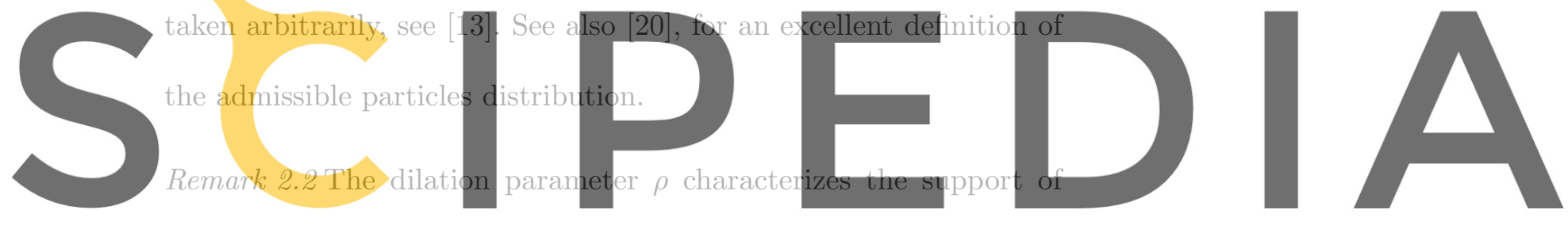

the shape functions $N_{i}^{\rho}(x)$. In fact, $\rho$ plays a role similar to the eleRegister for free at https//www.scipedia.com to download the version without the watermark

ment size $h$ in the finite element method. An enrichment similar to

$h$-refinement in finite elements can be produced in mesh-less methods decreasing the value of $\rho$ (this usually implies an increase in the number of particles). In the context of EFG, convergence properties depend on $m$ and $\rho$. The corresponding expressions do not depend explicitly on the distance between particles because usually, see [8,20], this distance is proportional to $\rho$, i.e. the ratio between the particle distance over the dilation parameter is kept constant. 
More precisely, in the remainder of the paper the following hypothesis is assumed to hold.

Hypothesis 2.1 In the refinement process, that is as $\rho$ goes to 0 , the particle distribution is homothetically densified. This is done ensuring that the particles belonging to a neighborhood of $x$ of radius $\rho$ keep the same pattern during the refinement. Moreover, this pattern is such that the matrix $\mathbf{M}$ is regular.

In order to clarify this hypothesis the following notation is introduced. Let $I_{x}^{\rho}$ be, for every $x \in \Omega$, the set of indices of the particles in the
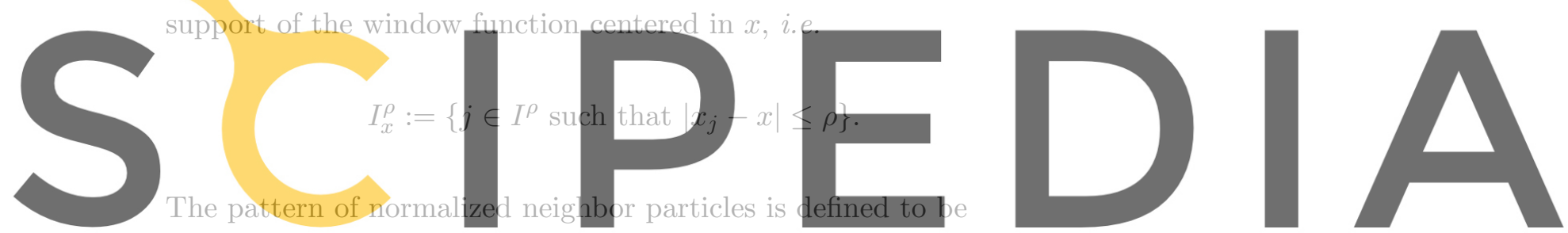

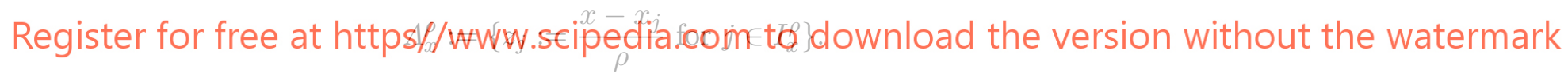

Thus, Hypothesis 2.1 is equivalent to assume that, passing from $\rho=\hat{\rho}$

to $\rho=\tilde{\rho}$, for every $\tilde{x} \in \Omega$ it exists $\hat{x} \in \Omega$ such that $\Lambda_{\tilde{x}}^{\tilde{\rho}}=\Lambda_{\hat{x}}^{\hat{\rho}}$. Consequently, $\left.\mathbf{M}(\hat{x})\right|_{\rho=\hat{\rho}}=\left.\mathbf{M}(\tilde{x})\right|_{\rho=\tilde{\rho}}$ and the properties of $\mathbf{M}$ are independent of $\rho$.

Remark 2.3 In Hypothesis 2.1 it is assumed that the distribution of particles in the neighborhood of any point $x$ is such that $\mathbf{M}(x)$ is regular. This condition is ensured having enough particles in the neigh- 
borhood of every point $x$ and located avoiding degenerated patterns, that is,

(i) $\operatorname{card} I_{x}^{\rho} \geq l+1$.

(ii) $\nexists F \in \operatorname{span}\left\{p_{0}, p_{1}, \ldots, p_{l}\right\} \backslash\{0\}$ such that $F\left(x_{i}\right)=0 \forall i \in I_{x}^{\rho}$ or, equivalently, for $F$ in $\operatorname{span}\left\{p_{0}, p_{1}, \ldots, p_{l}\right\}$

$\left[F\left(z_{i}\right)=0 \forall z_{i} \in \Lambda_{x}^{\rho} \Rightarrow F \equiv 0\right]$

Condition (ii) is easily verified. For instance, for $m=1$ (linear inter-

polation) the particles cannot lay in the same straight line or plane for, respectively, $2 \mathrm{D}$ and $3 \mathrm{D}$. In $1 \mathrm{D}$, for any value of $m$, it suffices

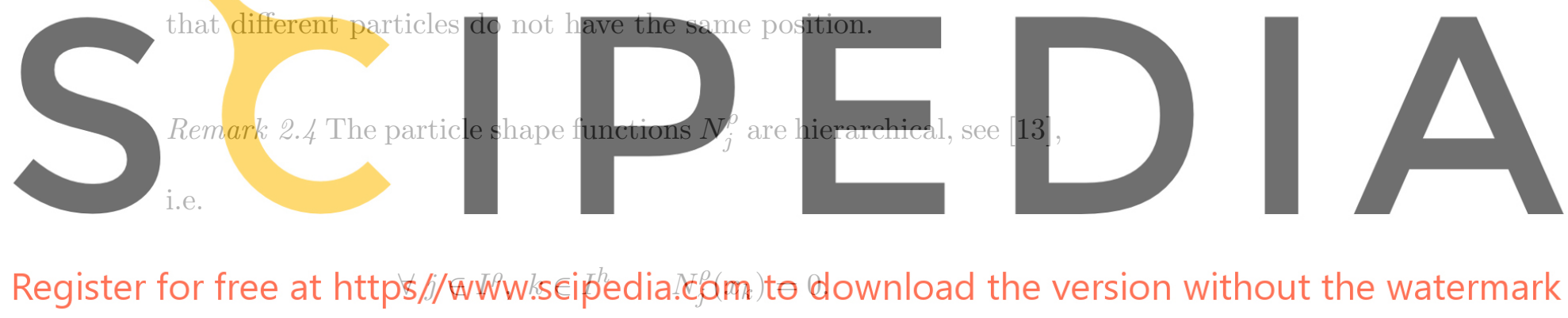

\section{Convergence analysis}

In the previous section a method for mixing $\mathrm{FE}$ and particles is introduced. This method allows to enrich the FE discretization everywhere adding particles and increasing the order of consistency. Recall that $p$ is the degree of the finite element interpolation, and $m$ is the order of consistency obtained with the added particles. Thus, the increment 
of consistency $q$ is such that

$$
q:=m-p .
$$

In this section an a priori error estimate for the proposed method is presented. First, two results of EFG (Lemma 3.1 and Lemma 3.2), also valid for the interpolation proposed here, are recalled. A proof of Lemma 3.1 can be found in [20]. Although a result similar to Lemma 3.2 may also be found in [20], here a proof of Lemma 3.2 is presented. Second, some properties of the FE basis are presented. Finally, the convergence of the combined interpolation is proved (Theorem 3.2).

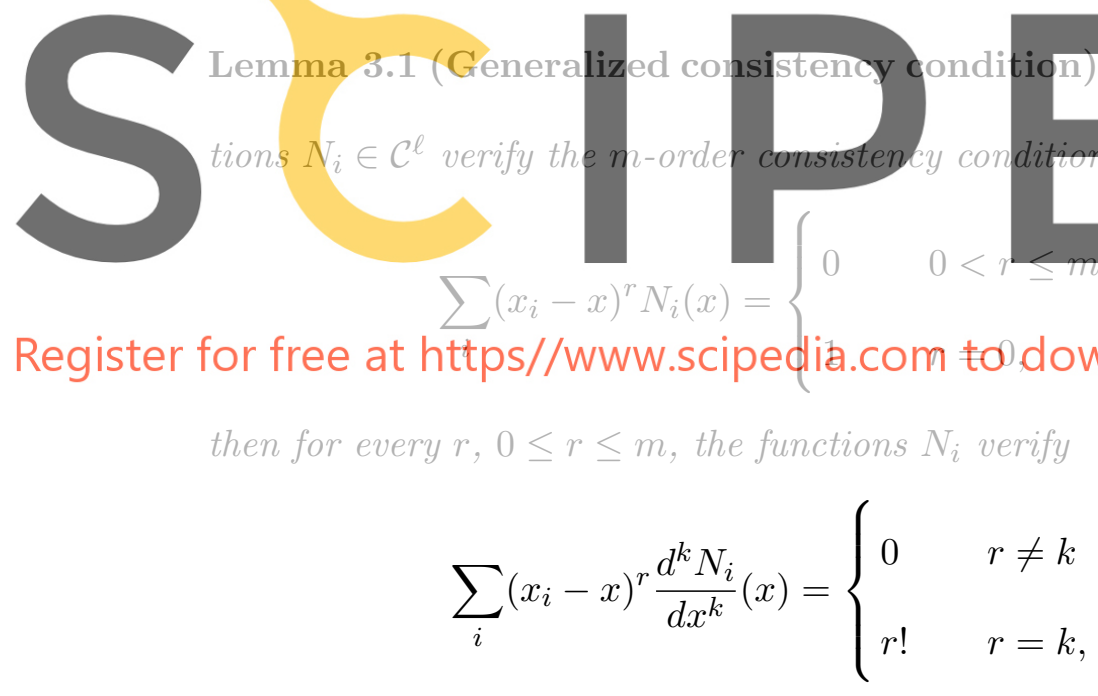

for all $k$ such that $0 \leq k \leq \ell$.

Lemma 3.2 Let $\Omega$ be an open bounded domain and $\phi \in \mathcal{C}^{\ell}(\Omega), \ell \leq$ $m$. Then, for all $x \in \Omega$, every element of matrix $\mathbf{M}^{-1}(x)$, the inverse of $\mathbf{M}(x)$, see (2.8), is bounded by a constant independent of $x$ and 
$\rho$. Moreover, the $k$-th derivative $(k \leq \ell)$ of every element of $\mathbf{M}$ is $\mathcal{O}\left(\rho^{-k}\right)$

Proof The first part of the Lemma, i.e. $\mathbf{M}^{-1}(x)$ is bounded, is evident

from Hypothesis 2.1. The $k$-th derivative of $\mathbf{M}(x)$ is rearranged using the normalized variable $z$ :

$$
\begin{aligned}
\frac{d^{k}}{d x^{k}} \mathbf{M}(x) & =\sum_{j \in I^{\rho}} \frac{d^{k}}{d x^{k}}\left[\mathbb{P}\left(\frac{x-x_{j}}{\rho}\right) \mathbb{P}^{T}\left(\frac{x-x_{j}}{\rho}\right) \phi\left(\frac{x-x_{j}}{\rho}\right)\right] \\
& =\left.\rho^{-k} \sum_{j \in I_{x}^{\rho}} \frac{d^{k}}{d z^{k}}\left[\mathbb{P}(z) \mathbb{P}^{T}(z) \phi(z)\right]\right|_{z=\left(x-x_{j}\right) / \rho}
\end{aligned}
$$

Under the assumption of Hypothesis 2.1 the term
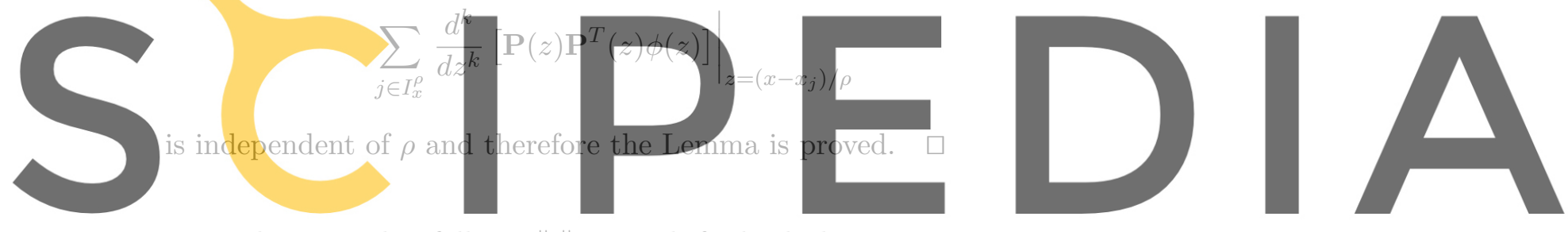

Remark 3.1 In what follows, $\|\cdot\|_{\infty}$ stands for both the maximum norm

Register for free at https//www.scipedia.com to download the version without the watermark

of a vector and the subordinate matrix norm ("max row sum"). Note

that the standard norm denoted by $\|\cdot\|_{\mathcal{L}_{\infty}}$, associated with the linear

space $\mathcal{L}_{\infty}$, is also used later.

Corollary 3.1 Under the assumptions of Lemma 3.2, constants $C^{\mathbf{M}}$ and $C_{k}^{\mathrm{M}}(k=0, \ldots, \ell)$ independent of $x$ and $\rho$, exist and are such that

$$
\left\|\mathbf{M}^{-1}(x)\right\|_{\infty} \leq C^{\mathbf{M}}, \quad \text { and } \quad\left\|\frac{d^{k} \mathbf{M}}{d x^{k}}\right\|_{\infty} \leq C_{k}^{\mathbf{M}} \rho^{-k}, \quad k=0, \ldots, \ell
$$


Proof Trivial given the definition of the matrix norm subordinate to the maximum vector norm.

Definition 3.1 Let $R_{\ell}^{(k)}(x), 0 \leq k \leq p$, be the $k$-th derivative of the remainder (pointwise error) in the finite element interpolation of the monomial $x^{\ell}$ :

$$
R_{\ell}^{(k)}(x):=\frac{d^{k}}{d x^{k}}\left[x^{\ell}-\sum_{i \in I^{h}} x_{i}^{\ell} N_{i}^{h}(x)\right]=\frac{d^{k}\left(x^{\ell}\right)}{d x^{k}}-\sum_{i \in I^{h}} x_{i}^{\ell} \frac{d^{k} N_{i}^{h}}{d x^{k}}(x) .
$$

In order to simplify the notation, the explicit dependence of $R_{\ell}^{(k)}$ on $x$ is omitted. If finite elements of order $p$ are used, in each element $R_{\ell}^{(k)}$ can be rewritten [14] as:

$$
R_{\ell}^{(k)}=\left\{\begin{array}{cc}
0 & 0 \leq \ell \leq p \\
\frac{\ell ! L^{k}(x) \xi^{\ell-(p+1)}}{(\ell-(p+1)) !(p+1-k) !} & p<\ell \leq m
\end{array}\right.
$$

where $\xi=\xi(x)$ is an unknown point inside the finite element where $x$ is located, where

$$
L^{k}(x)=\prod_{j=0}^{p-k}\left(x-\eta_{j}\right)
$$

and the $p+1-k$ distinct and unknown points, $\eta_{j}$, lie inside the finite element.

Lemma 3.3 For $k=0, \ldots, p$, the $p$-order finite element basis verifies

$$
\sum_{i \in I^{h}}\left(x-x_{i}\right)^{r} \frac{d^{k} N_{i}^{h}}{d x^{k}}(x)=0, \quad \text { for } r=1, \ldots, p \text { and } r>k
$$


and

$$
\left|\sum_{i \in I^{h}}\left(x-x_{i}\right)^{r} \frac{d^{k} N_{i}^{h}}{d x^{k}}(x)\right| \leq \mu_{r, k} h^{r-k}, \quad \text { for } r \geq p+1>k,
$$

where

$$
\mu_{r, k}:=\frac{r !}{(r-(p+1)) !(p+1-k) !} .
$$

Proof The left-hand side. of (3.4) is rearranged using the Newton's binomial expression:

$$
\begin{aligned}
\sum_{i \in I^{h}}\left(x-x_{i}\right)^{r} \frac{d^{k} N_{i}^{h}}{d x^{k}}(x) & =\sum_{i \in I^{h}}\left[\sum_{\ell=0}^{r}(-1)^{\ell}\left(\begin{array}{l}
r \\
\ell
\end{array} x_{i}^{\ell} x^{r-\ell}\right] \frac{d^{k} N_{i}^{h}}{d x^{k}}(x)\right. \\
& =\sum_{\ell=0}^{r}(-1)^{\ell}\left(\begin{array}{l}
r \\
\ell
\end{array} x^{r-\ell}\left[\sum_{i \in I^{h}} x_{i}^{\ell} \frac{d^{k} N_{i}^{h}}{d x^{k}}(x)\right]\right. \\
& =\sum_{\ell=0}^{r}(-1)^{\ell}\left(\begin{array}{l}
r \\
\ell
\end{array} x^{r-\ell}\left[\frac{d^{k}}{d x^{k}}\left(x^{\ell}\right)-R_{\ell}^{(k)}\right] .\right.
\end{aligned}
$$

Moreover,

$$
\frac{d^{k}}{d x^{k}}\left(x^{\ell}\right)=\left\{\begin{array}{cr}
0 & \text { if } \ell<k \\
\frac{\ell !}{(\ell-k) !} x^{\ell-k} & \text { if } \ell \geq k
\end{array}\right.
$$

and therefore $x^{r-\ell} \frac{d^{k}}{d x^{k}}\left(x^{\ell}\right)$ is either 0 for $\ell<k$, or $x^{r-k} \frac{\ell !}{(\ell-k) !}$ for $\ell \geq k$. Thus, using (3.2), (3.6) is expressed as

$$
\sum_{i \in I^{h}}\left(x-x_{i}\right)^{r} \frac{d^{k} N_{i}^{h}}{d x^{k}}(x)=x^{r-k} \sum_{\ell=k}^{r}\left(\begin{array}{l}
r \\
\ell
\end{array}\right) \frac{(-1)^{\ell} \ell !}{(\ell-k) !}-\sum_{\ell=p+1}^{r}(-1)^{\ell}\left(\begin{array}{l}
r \\
\ell
\end{array}\right) x^{r-\ell} R_{\ell}^{(k)} .
$$


Note that the first sum of the right-hand side. term of (3.8) cancels because, for $r>k$,

$$
\sum_{\ell=k}^{r}(-1)^{\ell}\left(\begin{array}{l}
r \\
\ell
\end{array}\right) \frac{\ell !}{(\ell-k) !}=(-1)^{k} \frac{r !}{(r-k) !}\left[\sum_{j=0}^{r-k}(-1)^{j}\left(\begin{array}{c}
r-k \\
j
\end{array}\right)\right]=0,
$$

and consequently

$$
\sum_{i \in I^{h}}\left(x-x_{i}\right)^{r} \frac{d^{k} N_{i}^{h}}{d x^{k}}(x)=\sum_{\ell=p+1}^{r}(-1)^{\ell+1}\left(\begin{array}{l}
r \\
\ell
\end{array}\right) x^{r-\ell} R_{\ell}^{(k)}
$$

If $r \leq p$, obviously $\ell$ cannot range between $p+1$ and $r$, thus,

$$
\sum_{i \in I^{h}}\left(x-x_{i}\right)^{r} \frac{d^{k} N_{i}^{h}}{d x^{k}}(x)=0, \quad r \leq p .
$$

which does coincide with (3.4). For $r>p$, using (3.2), (3.10) becomes

$$
\sum_{i \in I^{h}}\left(x-x_{i}\right)^{r} \frac{d^{k} N_{i}^{h}}{d x^{k}}(x)=\frac{L^{k}(x) r !}{(p+1-k) !} \sum_{\ell=p+1}^{r} \frac{(-1)^{\ell+1} x^{r-\ell} \xi^{\ell-(p+1)}}{(\ell-(p+1)) !(r-\ell) !}
$$

A new mute index $j:=\ell-(p+1)$ and a new parameter $s:=r-(p+1)$ are defined and thus, (3.11) can be rewritten as

$$
\begin{aligned}
\sum_{i \in I^{h}}\left(x-x_{i}\right)^{r} \frac{d^{k} N_{i}^{h}}{d x^{k}}(x) & =(-1)^{p} \frac{L^{k}(x) r !}{(p+1-k) ! s !} \sum_{j=0}^{s}\left(\begin{array}{l}
s \\
j
\end{array}\right) x^{s-j}(-\xi)^{j} \\
& =(-1)^{p} \frac{L^{k}(x) r !}{(p+1-k) ! s !}(x-\xi)^{s} .
\end{aligned}
$$


Since $\left|L^{k}(x)\right| \leq h^{p+1-k}$, when $|x-\xi| \leq h$, (3.12) can be bounded, namely,

$$
\left|\sum_{i \in I^{h}}\left(x-x_{i}\right)^{r} \frac{d^{k} N_{i}^{h}}{d x^{k}}(x)\right| \leq \frac{r !}{(p+1-k) ! s !} h^{r-k},
$$

which is precisely the inequality (3.5).

Remark 3.2 The term that cancels according to (3.9), that is the first sum of the right-hand side. term of (3.8), is a rearranged expression of

$$
\sum_{\ell=0}^{r}(-1)^{\ell}\left(\begin{array}{l}
r \\
\ell
\end{array}\right) x^{r-\ell} \frac{d^{k}}{d x^{k}}\left(x^{\ell}\right)
$$

for $r>k$, see (3.6). However, for $k>r$, this term is obviously also zero because $d^{k}\left(x^{\ell}\right) / d x^{k}=0$ for $\ell=0, \ldots, r$. This term is not zero for $k=r$. In this case, the cited term takes the value of $(-1)^{r} r$ !. Nevertheless, Lemma 3.3 is restricted to $r>k$ because it is the only case needed in the rest of the paper.

In order to prove the convergence results (error bound theorems) several lemmas and a theorem are needed. Their goal is to bound the shape function, $N_{j}^{\rho}$, and its derivatives. Hence, a bound on the right-hand side. of (2.7) and then a bound for $\boldsymbol{\alpha}$ are needed.

Lemma 3.4 Let $q_{r}(x):=x^{r}$. Then, for $k=0, \ldots, p$,

$$
\frac{d^{k}}{d x^{k}}\left[q_{r}(0)-\sum_{i \in I^{h}} q_{r}\left(\frac{x-x_{i}}{\rho}\right) N_{i}^{h}(x)\right]=0, \quad r=0, \ldots, p
$$


and

$$
\left|\frac{d^{k}}{d x^{k}}\left[q_{r}(0)-\sum_{i \in I^{h}} q_{r}\left(\frac{x-x_{i}}{\rho}\right) N_{i}^{h}(x)\right]\right| \leq \lambda_{k, r} \frac{h^{r-k}}{\rho^{r}}, \quad r \geq p+1,
$$

where $\lambda_{k, r}$ are the following constants independent of $x, \rho$ and $h$ :

$$
\lambda_{k, r}:=\frac{r !}{(r-k) !} \sum_{\ell=\max \{k-r+p+1,0\}}^{k}\left(\begin{array}{l}
k \\
\ell
\end{array}\right)\left(\begin{array}{c}
r-k \\
p+1-\ell
\end{array}\right) .
$$

Proof In order to simplify the notation

$$
t_{r}(x):=q_{r}(0)-\sum_{i \in I^{h}} q_{r}\left(\frac{x-x_{i}}{\rho}\right) N_{i}^{h}(x)
$$

is defined. Three cases are considered: $r=0,1 \leq r \leq p$ and $p<r$. In the first case, for $r=0, q_{0}(x)=1$ and $t_{0}(x)=1-\sum_{i \in I^{h}} N_{i}^{h}(x)=$ $1-1=0$. In the second case, for $r=1, \ldots, p$, Lemma 3.3 gives, for $k=0$,

$$
t_{r}(x)=-\frac{1}{\rho^{r}}\left[\sum_{i \in I^{h}}\left(x-x_{i}\right)^{r} N_{i}^{h}(x)\right]=0 .
$$

Hence, $d^{k}\left(t_{r}(x)\right) / d x^{k}=0$ for every $k$ and, consequently, (3.14) is proved. For the last case, $r>p$,

$$
\begin{aligned}
\frac{d^{k} t_{r}}{d x^{k}}(x) & =-\frac{1}{\rho^{r}} \sum_{i \in I^{h}} \frac{d^{k}}{d x^{k}}\left[\left(x-x_{i}\right)^{r} N_{i}^{h}(x)\right] \\
& =-\frac{1}{\rho^{r}} \sum_{i \in I^{h}}\left\{\sum_{\ell=0}^{k}\left(\begin{array}{l}
k \\
\ell
\end{array}\right) \frac{d^{k-\ell}}{d x^{k-\ell}}\left[\left(x-x_{i}\right)^{r}\right] \frac{d^{\ell} N_{i}^{h}}{d x^{\ell}}(x)\right\} .
\end{aligned}
$$


Using (3.7) and changing the order of the sums, the previous formula is expressed as

$$
\frac{d^{k} t_{r}}{d x^{k}}(x)=-\frac{1}{\rho^{r}} \sum_{\ell=0}^{k}\left(\begin{array}{l}
k \\
\ell
\end{array}\right) \frac{r !}{(r-k+\ell) !}\left[\sum_{i \in I^{h}}\left(x-x_{i}\right)^{r-k+\ell} \frac{d^{\ell} N_{i}^{h}}{d x^{\ell}}(x)\right] .
$$

Note that $r-k+\ell>\ell$ because $r>p \geq k$. Then, Lemma 3.3 applies and the sum with index $i$ is zero if $\ell+r-k \leq p$. Therefore, the sum in $\ell$ begins with $\ell=\max \{k-r+(p+1), 0\}$. Using now Lemma 3.3 for the remaining terms, the bound (3.15) is proved:

$\left|\frac{d^{k} t_{r}}{d x^{k}}(x)\right| \leq \frac{1}{\rho^{r}}\left[\sum_{\ell=\max \{k-r+p+1,0\}}^{k}\left(\begin{array}{l}k \\ \ell\end{array}\right) \frac{r !}{(r-k+\ell) !} \mu_{r-k+\ell, \ell}\right] h^{r-k} . \square$

Lemma 3.5 Let $k$ be such that $0 \leq k \leq p$. Suppose $h \leq Q_{k} \rho$, where the following definition $Q_{k}$ stands for given $p$ and $m$ :

$$
Q_{k}:= \begin{cases}1 & \text { for } m=p+1, \\ \min _{r=p+2, \ldots, m}\left(\frac{\lambda_{k, p+1}}{\lambda_{k, r}}\right)^{\frac{1}{r-(p+1)}} & \text { for } m>p+1 .\end{cases}
$$

and $\lambda_{k, r}$ are the constants defined in (3.16). Then, the derivatives of the right-hand side. of equation (2.7) verify the following inequality:

$$
\left\|\frac{d^{k}}{d x^{k}}\left[\mathbf{P}(0)-\sum_{i \in I^{h}} \mathbf{P}\left(\frac{x-x_{i}}{\rho}\right) N_{i}^{h}(x)\right]\right\|_{\infty} \leq \lambda_{k, p+1} \frac{h^{p+1-k}}{\rho^{p+1}} .
$$

Proof Since $h \leq Q_{k} \rho$, by definition (3.18), then for $r=p+2, \ldots, m$

$$
\lambda_{k, r}\left[\frac{h}{\rho}\right]^{r-(p+1)} \leq \lambda_{k, p+1} .
$$


Using now Lemma 3.4 for every component, $t_{r}(x)$, of the right-hand side. in (2.7) one gets

$$
\begin{gathered}
\frac{d^{k} t_{r}}{d x^{k}}=0 \quad \text { for } r=0, \ldots, p, \\
\frac{d^{k} t_{r}}{d x^{k}}=\lambda_{k, p+1} \frac{h^{p+1-k}}{\rho^{p+1}} \quad \text { for } r=p+1,
\end{gathered}
$$

and

$\left|\frac{d^{k} t_{r}}{d x^{k}}(x)\right| \leq \lambda_{k, r}\left[\frac{h}{\rho}\right]^{r-(p+1)} \frac{h^{p+1-k}}{\rho^{p+1}} \leq \lambda_{k, p+1} \frac{h^{p+1-k}}{\rho^{p+1}} \quad$ for $r=p+2, \ldots, m$.

Lemma 3.6 Let $k$ be such that $0 \leq k \leq p$. Suppose $h / \rho \leq \min _{0 \leq s \leq k} Q_{s}$, where $Q_{s}$ is the constant defined in (3.18). Then, it exists a constant $C_{k}^{\star}$, independent of $\rho, h$ and $x$, such that the solution, $\boldsymbol{\alpha}$, of the linear system of equations (2.7) verifies

$$
\left\|\frac{d^{k} \boldsymbol{\alpha}}{d x^{k}}\right\|_{\infty} \leq C_{k}^{\star} \frac{h^{p+1-k}}{\rho^{p+1}} .
$$

Proof The right-hand side. of (2.7) is defined as $\mathbf{T}=\mathbf{T}(x):=\mathbf{P}(0)-$ $\sum_{i \in I^{h}} \mathbf{P}\left(\frac{x-x_{i}}{\rho}\right) N_{i}^{h}(x)$. Thus, equation (2.7) is rewritten as

$$
\mathbf{M} \alpha=\mathbf{T}
$$

Using Lemma 3.5 and Corollary 3.1, the previous equation implies:

$$
\|\boldsymbol{\alpha}\|_{\infty} \leq\left\|\mathbf{M}^{-1}\right\|_{\infty}\|\mathbf{T}\|_{\infty} \leq \underbrace{C^{\mathbf{M}} \lambda_{0, p+1}}_{C_{0}^{\star}} \frac{h^{p+1}}{\rho^{p+1}} .
$$

Once (3.20) is proved for $k=0$, we proceed by induction on $k$, that is, assume

$$
\left\|\frac{d^{s} \boldsymbol{\alpha}}{d x^{s}}\right\|_{\infty} \leq C_{s}^{\star} \frac{h^{p+1-s}}{\rho^{p+1}} \quad \text { for } s=0, \ldots, k-1 .
$$


Differentiating (3.21) yields

$$
\left[\sum_{s=0}^{k-1}\left(\begin{array}{l}
k \\
s
\end{array}\right) \frac{d^{k-s} \mathbf{M}}{d x^{k-s}} \frac{d^{s} \boldsymbol{\alpha}}{d x^{s}}\right]+\mathbf{M} \frac{d^{k} \boldsymbol{\alpha}}{d x^{k}}=\frac{d^{k} \mathbf{T}}{d x^{k}} .
$$

Rearranging terms, the following expression for the $k$-th derivative of $\boldsymbol{\alpha}$ is found:

$$
\frac{d^{k} \boldsymbol{\alpha}}{d x^{k}}=\mathbf{M}^{-1}\left[\frac{d^{k} \mathbf{T}}{d x^{k}}-\sum_{s=0}^{k-1}\left(\begin{array}{l}
k \\
s
\end{array}\right) \frac{d^{k-s} \mathbf{M}}{d x^{k-s}} \frac{d^{s} \boldsymbol{\alpha}}{d x^{s}}\right]
$$

Since $\rho / h \leq Q_{k}$, using Lemma 3.5, Corollary 3.1 and the induction hypothesis (3.22), the following inequality is obtained:

$$
\begin{aligned}
\left\|\frac{d^{k} \boldsymbol{\alpha}}{d x^{k}}\right\|_{\infty} & \leq\left\|\mathbf{M}^{-1}\right\|_{\infty}\left[\left\|\frac{d^{k} \mathbf{T}}{d x^{k}}\right\|_{\infty}+\sum_{s=0}^{k-1}(k)\left\|\frac{d^{k-s} \mathbf{M}}{d x^{k-s}}\right\|_{\infty}\left\|\frac{d^{s} \boldsymbol{\alpha}}{d x^{s}}\right\|_{\infty}\right] \\
& \leq C^{\mathbf{M}}\left[\lambda_{k, p+1} \frac{h^{p+1-k}}{\rho^{p+1}}+\sum_{s=0}^{k-1}(k) C_{k-s}^{\mathbf{M}} \rho^{s-k} C_{s}^{\star} \frac{h^{p+1-s}}{\rho^{p+1}}\right] \\
& \leq \widehat{C} \frac{h^{p+1-k}}{\rho^{p+1}}+\sum_{s=0}^{k-1} \widehat{C}_{s} \frac{h^{p+1-s}}{\rho^{p+1+k-s}} .
\end{aligned}
$$

When $k>0$, assuming $h / \rho \leq Q_{s}$ for $s=0, \ldots, k-1$, it follows that

$$
\frac{h^{p+1-s}}{\rho^{p+1+k-s}} \leq\left(Q_{s}\right)^{k-s} \frac{h^{p+1-k}}{\rho^{p+1}} .
$$

Consequently,

$$
\left\|\frac{d^{k} \boldsymbol{\alpha}}{d x^{k}}\right\|_{\infty} \leq C_{k}^{\star} \frac{h^{p+1-k}}{\rho^{p+1}} . \square
$$

Theorem 3.1 (Shape function's bound) Let $m$ be the order of consistency of the combined approximation $u^{h}+u^{\rho}$, such that $m=$ 
$p+q$, where $p$ is the order of the finite element approximation, $u^{h}$, and $q>0$ is the order increment due to $u^{\rho}$. Let $\ell$ be such that $0 \leq \ell \leq p$. Assume the following regularity conditions for the exact solution, $u$, and the weighting function, $\phi: u \in \mathcal{C}^{m+1}(\bar{\Omega})$ and $\phi \in \mathcal{C}^{\ell}(\bar{\Omega})$, where $\Omega$ is bounded and $\partial \Omega$ is smooth. Finally, assume that $h / \rho \leq \min _{0 \leq k \leq \ell} Q_{k}$ where $Q_{k}$ is the constant defined in (3.18). Then, for $k=0, \ldots, \ell$, the following inequality holds:

$$
\left\|\frac{d^{k} N_{j}^{\rho}}{d x^{k}}\right\|_{L^{\infty}} \leq C_{k}^{\star \star} \frac{h^{p+1-k}}{\rho^{p+1}},
$$

where $C_{k}^{\star \star}$ is a constant independent of $x, \rho$ and $h$.

Proof Using the Newton's binomial expression in (2.4), the $k$-th derivative of the shape function $N_{j}^{\rho}$ is

$$
\frac{d^{k} N_{j}^{\rho}}{d x^{k}}(x)=\left.\sum_{s=0}^{k}(k)\left[\frac{d^{s} \boldsymbol{\alpha}}{d x^{s}}(x)\right]^{T} \frac{1}{\rho^{k-s}} \frac{d^{k-s}[\mathbf{P}(z) \phi(z)]}{d z^{k-s}}\right|_{z=\left(x-x_{i}\right) / \rho}
$$

Note that $\phi(z)=0$ if $|z|>1$. Therefore, by Lemma 3.6,

$$
\left|\frac{d^{k} N_{j}^{\rho}}{d x^{k}}(x)\right| \leq \sum_{s=0}^{k}\left(\begin{array}{l}
k \\
s
\end{array}\right) C_{s}^{\star} \frac{h^{p+1-s}}{\rho^{p+1+k-s}}\left\{\max _{|z| \leq 1}\left\|\frac{d^{k-s}[\mathbf{P}(z) \phi(z)]}{d z^{k-s}}\right\|_{\infty}\right\}
$$

Note that the term in braces is a constant independent of $h$ and $\rho$. Moreover, with the assumption $h / \rho \leq \min _{0 \leq k \leq \ell} Q_{k}$, see equation (3.23), 
it follows that

$$
\left|\frac{d^{k} N_{j}^{\rho}}{d x^{k}}(x)\right| \leq \underbrace{\left[\sum_{s=0}^{k}(k) C_{s}^{\star}\left(Q_{s}\right)^{k-s}\left\{\max _{|z| \leq 1}\left\|\frac{d^{k-s}[\mathbf{P}(z) \phi(z)]}{d z^{k-s}}\right\|_{\infty}\right\}\right]}_{C_{k}^{\star \star}} \frac{h^{p+1-k}}{\rho^{p+1}} . \square
$$

Theorem 3.2 (Error bound) Under the same assumptions of The-

orem 3.1,

$$
\left|u-\left(u^{h}+u^{\rho}\right)\right|_{\mathcal{W}_{\infty}^{k}} \leq h^{p+1-k}\left[C_{1} h^{q}+C_{2} \rho^{q}\right]|u|_{\mathcal{W}_{\infty}^{m+1}} \quad k=0, \ldots, \ell
$$

where $C_{1}$ and $C_{2}$ are independent of the finite element size, $h$, and the dilation parameter, $\rho$, of the mesh-less approximation.

Proof Let $x$ be a point in $\Omega$ and let $x_{i}$ be either a node of the finite element where $x$ is located, i.e. $\left|x-x_{i}\right|<h$, or a particle such that $N_{i}^{\rho}(x) \neq 0$, i.e. $\left|x-x_{i}\right|<\rho$. Consider the $m$-order Taylor expansion of $u$ around $x$

$$
\begin{array}{r}
u\left(x_{i}\right)=u(x)+\left(x_{i}-x\right) \frac{d u}{d x}(x)+\frac{\left(x_{i}-x\right)^{2}}{2} \frac{d^{2} u}{d x^{2}}(x)+\cdots \\
+\frac{\left(x_{i}-x\right)^{m}}{m !} \frac{d^{m} u}{d x^{m}}(x)+\frac{\left(x_{i}-x\right)^{m+1}}{(m+1) !} \frac{d^{m+1} u}{d x^{m+1}}(\xi),
\end{array}
$$

where $\xi=\theta x+(1-\theta) x_{i}$, for some $\theta$ such that $0 \leq \theta \leq 1$. The point $\xi$ lies in $\Omega$ if the finite elements are convex and $\rho$ is small enough near the smooth boundary. Let us define

$$
N_{i}(x):= \begin{cases}N_{i}^{h}(x) & i \in I^{h}, \\ N_{i}^{\rho}(x) & i \in I^{\rho} .\end{cases}
$$


The derivative of the approximation of $u$ yields

$$
\frac{d^{k} u}{d x^{k}}(x) \simeq \frac{d^{k}\left(u^{h}+u^{\rho}\right)}{d x^{k}}(x)=\sum_{i \in I^{h} \cup I^{\rho}} u\left(x_{i}\right) \frac{d^{k} N_{i}}{d x^{k}}(x) .
$$

The shape functions $N_{j}^{\rho}$ are derived in order to achieve $m$-order consistency. Thus, the complete set of functions $N_{i}$ (including the finite element basis) has $m$-order consistency. Then, replacing (3.26) in (3.27), and applying Lemma 3.1, a new expression is obtained:

$$
\begin{aligned}
\frac{d^{k}\left(u^{h}+u^{\rho}\right)}{d x^{k}}(x)= & u(x) \underbrace{\sum_{i \in I^{h} \cup I^{\rho}} \frac{d^{k} N_{i}}{d x^{k}}(x)}_{0}+\frac{d u}{d x}(x) \underbrace{\sum_{i \in I^{h} \cup I^{\rho}}\left(x_{i}-x\right) \frac{d^{k} N_{i}}{d x^{k}}(x)}_{0}+\cdots \\
& +\frac{1}{k !} \frac{d^{k} u}{d x^{k}}(x) \underbrace{\sum_{i \in I^{h} \cup I^{\rho}}\left(x_{i}-x\right)^{k} \frac{d^{k} N_{i}}{d x^{k}}(x)}_{k !}+\cdots \\
& +\frac{1}{m !} \frac{d^{m} u}{d x^{m}}(x) \underbrace{\sum_{i \in I^{h} \cup I^{\rho}}\left(x_{i}-x\right)^{m} \frac{d^{k} N_{i}}{d x^{k}}(x)}_{0} \\
& +\frac{1}{(m+1) !} \frac{d^{m+1} u}{d x^{m+1}(\xi) \sum_{i \in I^{h} \cup I^{\rho}}\left(x_{i}-x\right)^{m+1}} \frac{d^{k} N_{i}}{d x^{k}}(x) .
\end{aligned}
$$

The previous expression can be rewritten as:

$$
\begin{gathered}
\frac{d^{k}\left[u-\left(u^{h}+u^{\rho}\right)\right]}{d x^{k}}(x)=\frac{-1}{(m+1) !} \frac{d^{m+1} u}{d x^{m+1}}(\xi)\left[\sum_{i \in I^{h}}\left(x_{i}-x\right)^{m+1} \frac{d^{k} N_{i}^{h}}{d x^{k}}(x)\right. \\
\left.+\sum_{j \in I^{\rho}}\left(x_{j}-x\right)^{m+1} \frac{d^{k} N_{j}^{\rho}}{d x^{k}}(x)\right] .
\end{gathered}
$$

For a given $x$ the first sum in the right-hand side. of (3.28) may be restricted to the $p+1$ nodes of the finite element where $x$ is 
located and which verify $\left|x-x_{i}\right| \leq h$. Similarly, the second sum in the right-hand side. of (3.28) is circumscribed to the particles $x_{j}$ such that $x$ is included in the support of $N_{j}^{\rho}(x)$, i.e. particles such that $\left|x-x_{j}\right| \leq \rho$. Let us denote by $n$ the maximum number of particles verifying $\left|x-x_{j}\right| \leq \rho$. Hence, from (3.28) the following bound is obtained

$$
\begin{aligned}
\left|\frac{d^{k}\left(u^{\rho}+u^{h}\right)}{d x^{k}}(x)\right| \leq \frac{1}{(m+1) !} & \left|\frac{d^{m+1} u}{d x^{m+1}}(\xi)\right|\left[(p+1) h^{m+1} \max _{i \in I^{h}}\left|\frac{d^{k} N_{i}^{h}}{d x^{k}}\right|\right. \\
& \left.+n \rho^{m+1} \max _{j \in I^{\rho}}\left|\frac{d^{k} N_{j}^{\rho}}{d x^{k}}\right|\right] .
\end{aligned}
$$

On one hand, the $k$-th derivative $(k<p)$ of the finite element shape functions is $\mathcal{O}\left(h^{-k}\right)$. On the other, Theorem 3.1 bounds the shape functions $N_{j}^{\rho}$ and their derivatives. Consequently,

$$
\begin{aligned}
\left\|\frac{d^{k} u}{d x^{k}}-\frac{d^{k}\left(u^{\rho}+u^{h}\right)}{d x^{k}}\right\|_{\mathcal{L}_{\infty}} & \leq\left[C_{1} h^{m+1-k}+C_{2} \frac{h^{p+1-k}}{\rho^{p+1}} \rho^{m+1}\right]\left\|\frac{d^{k} u^{m+1}}{d x^{k}}\right\|_{\mathcal{L}_{\infty}} \\
& \leq h^{p+1-k}\left[C_{1} h^{q}+C_{2} \rho^{q}\right]\left\|\frac{d^{k} u^{m+1}}{d x^{k}}\right\|_{\mathcal{L}_{\infty}},
\end{aligned}
$$

which is precisely $(3.25)$.

Corollary 3.2 Let $m$ be the order of consistency of the combined approximation $u^{h}+u^{\rho}$, such that $m=p+q$, where $p$ is the order of the finite element approximation, $u^{h}$, and $q>0$ is the order increment due to $u^{\rho}$. Suppose that the following regularity conditions hold for the exact solution, $u$, and the weighting function, $\phi: u \in C^{m+1}(\bar{\Omega})$ and $\phi \in C^{0}(\bar{\Omega})$, where $\Omega$ is bounded and $\partial \Omega$ is smooth. Finally, assume 
that the element size $h$ is small enough with respect to the dilation parameter $\rho$, i.e.

$$
\frac{h}{\rho} \leq \min _{r=p+1, \ldots, m}\left(\begin{array}{c}
r \\
p+1
\end{array}\right)^{\frac{-1}{r-(p+1)}}
$$

Then,

$$
\left\|u-\left(u^{h}+u^{\rho}\right)\right\|_{\mathcal{L}_{\infty}} \leq h^{p+1}\left[C_{1} h^{q}+C_{2} \rho^{q}\right]|u|_{\mathcal{W}_{\infty}^{m+1}}
$$

where $C_{1}$ and $C_{2}$ are independent of the finite element size, $h$, and the dilation parameter, $\rho$, of the mesh-less approximation.

Remark 3.3 The error bound given in (3.29) allows to predict the convergence behavior of the combined finite element-particle interpolation. That is, when both $h$ and $\rho$ decrease simultaneously, the order of convergence is $p+q+1=m+1$. When $h$ goes to zero while $\rho$ is kept constant, the order is either $p+1$ if $C_{1} h^{q}<C_{2} \rho^{q}$ or $m+1$ when $C_{1} h^{q} \gg C_{2} \rho^{q}$. And finally, convergence is ensured at a rate of $q$ when $\rho$ goes to zero provided that $C_{1} h^{q} \ll C_{2} \rho^{q}$. But, if $h$ is kept constant as $\rho$ goes to zero, it is necessary to increase the order of consistency in order to achieve asymptotic convergence. Numerical examples of these situations are shown in [13].

If the restriction on the mesh size, $h / \rho \leq \min _{0 \leq k \leq \ell} Q_{k}$, is omitted, Lemma 3.5 must be rewritten, the previous results are no longer valid and must be replaced by less sharp error bounds. 
Lemma 3.7 With no restriction on the element size, the independent term in equation (2.7) verifies

$\left\|\frac{d^{k}}{d x^{k}}\left[\mathbf{P}(0)-\sum_{i \in I^{h}} \mathbf{P}\left(\frac{x-x_{i}}{\rho}\right) N_{i}^{h}(x)\right]\right\|_{\infty} \leq \lambda_{k, p+1} \frac{h^{p+1-k}}{\rho^{m}}, \quad k=0, \ldots, p$.

Proof Trivial from Lemma 3.4.

Reproducing the proof of the previous lemmas, the following less accurate error bound is easily proved:

Theorem 3.3 (Error bound) Under the same assumptions of Theorem 3.2 but with no restriction on the element size, the error bound $i s$

$$
\left|u-\left(u^{h}+u^{\rho}\right)\right|_{\mathcal{W}_{\infty}^{k}} \leq h^{p+1-k}\left[C_{1} h^{q}+C_{2} \rho^{\mathbf{1}}\right]|u|_{\mathcal{W}_{\infty}^{m+1}}
$$

where $C_{1}$ and $C_{2}$ are independent of the finite element size, $h$, and the dilation parameter, $\rho$, of the mesh-less approximation.

Corollary 3.3 Under the same assumptions of Corollary 3.2 but with no restriction on the element size, the following inequality holds:

$$
\left\|u-\left(u^{h}+u^{\rho}\right)\right\|_{\mathcal{L}_{\infty}} \leq h^{p+1}\left[C_{1} h^{q}+C_{2} \rho^{\mathbf{1}}\right]|u|_{\mathcal{W}_{\infty}^{m+1}}
$$

where $C_{1}$ and $C_{2}$ are independent of the finite element size, $h$, and the dilation parameter, $\rho$, of the mesh-less approximation. 


\section{References}

1. I. Babuška and J.M. Melenk. The partition of unity finite method. International Journal for Numerical Methods in Engineering, 40:727-758, 1997.

2. S. Beissel and T. Belytschko. Nodal integration of the element-free galerkin method. Computer Methods in Applied Mechanics and Engineering, 139:4974,1996

3. T. Belytschko, Y. Krongauz, D. Organ, M. Fleming, and P. Krysl. Meshless methods: an overview and recent developments. Computer Methods in Applied Mechanics and Engineering, 139:3-47, 1996.

4. T. Belytschko, Y. Y. Lu, and L. Gu. Element-free galerkin methods. International Journal for Numerical Methods in Engineering, 37:229-256, 1994.

5. T. Belytschko and D. Organ. Element-free galerkin methods for dynamic fracture in concrete. In D.R.J. Owen, E. O nate, and E. Hilton, editors, Computational Plasticity. Fundamentals and Applications, pages 304-321, 1997.

6. T. Belytschko, D. Organ, and Y. Krongauz. A coupled finite element-free galerkin method. Computational Mechanics, 17:186-195, 1995.

7. T. Belytschko and M. Tabbara. Dynamic fracture using element-free galerkin methods. International Journal for Numerical Methods in Engineering, 39:923-938, 1996.

8. B. Ben Moussa and J. P. Vila. Convergence of sph method for scalar nonlinear conservation laws. SIAM J. NUMER. ANAL, 1999. To appear.

9. J. Bonet and S. Kulasegaram. Correction and stabilization of smooth particle hydrodynamics methods with applications in metal forming simulations. International Journal for Numerical Methods in Engineering, 47:1189-1214, 2000. 
10. C. A. Duarte and J. T. Oden. An $h-p$ adaptive method using clouds. Computer Methods in Applied Mechanics and Engineering, 139:237-262, 1996.

11. C. A. Duarte and J. T. Oden. Hp clouds - an hp meshless method. Numerical Methods for Partial Differential Equations, 12:673-705, 1996.

12. D. Hegen. Element free galerkin methods in combination with finite element approaches. Computer Methods in Applied Mechanics and Engineering, 135:143-166, 1996.

13. A. Huerta and S. Fernández-Méndez. Enrichment and coupling of the finite element and meshless methods. International Journal for Numerical Methods in Engineering, 48:1615-1636, 2000.

14. E. Isaacson and H. B. Keller. Analysis of numerical methods. John Wiley \& Sons, New York, 1966. (Reprinted by Dover Publications, 1994).

15. P. Lancaster and K. Salkauskas. Surface generated by moving least square methods. Mathematics of Computation, 37(155):141-158, 1981.

16. T. J. Liszka, C. A. M. Duarte, and W. W. Tworzydlo. hp-meshless cloud method. Computer Methods in Applied Mechanics and Engineering, 139:263$288,1996$.

17. W. K. Liu and Y. Chen. Wavelet and multiple scale reproducing kernel methods. International Journal for Numerical Methods in Fluids, 21:901931, 1995.

18. W. K. Liu, Y. Chen, S. Jun, J. S. Chen, T. Belytschko, C. Pan, R. A. Uras, and C. T. Chang. Overview and applications of the reproducing kernel particle methods. Archives of Computational Methods in Engineering, State of the Art Reviews, 3:3-80, 1996.

19. W. K. Liu, S. Jun, and Y. F. Zhang. Reproducing kernel particle methods. International Journal for Numerical Methods in Fluids, 20:1081-1106, 1995. 
20. W. K. Liu, S. Li, and T. Belytschko. Moving least square reproducing kernel methods. (i) methodology and convergence. Computer Methods in Applied Mechanics and Engineering, 143:113-154, 1997.

21. W. K. Liu, R. A. Uras, and Y. Chen. Enrichment of the finite element method with reproducing kernel particle method. Journal of Applied Mechanics ASME, 64:861-870, 1997.

22. Y. Y. Lu, T. Belytschko, and L. Gu. A new implementation of the element free galerkin method. Computer Methods in Applied Mechanics and Engineering, $113: 397-414,1994$.

23. J. M. Melenk and I. Babuška. The partition of unity finite element method: basic theory and applications. Computer Methods in Applied Mechanics and Engineering, 139:289-314, 1996.

24. J. J. Monaghan. Why particle methods work. SIAM J. SCI. STAT. COMPUT., $3(4): 422-433,1982$.

25. D. Organ, M. Fleming, T. Terry, and T. Belytschko. Continuous meshless approximations for nonconvex bodies by diffraction and transparency. Computational Mechanics, 18:225-235, 1997.

26. J. P. Vila. On particle weighted methods and smooth particle hydrodynamics. Mathematical Models and Methods in Applied Sciences, 9(2):161-209, 1999. 